REDES. Revista hispana para el análisis de redes sociales.

Vol.4,\#5, jun-jul. 2003.

http://revista-redes.rediris.es

\title{
Las redes sociales como herramienta de análisis estructural input-output
}

\author{
Ana Salomé García Muñiz ${ }^{1}$ \\ Carmen Ramos Carvajal \\ Universidad de Oviedo- Departamento de Economía Aplicada
}

\begin{abstract}
Uno de los aspectos fundamentales que posibilita el conocimiento en profundidad de una economía es la realización de un análisis de su estructura productiva. Dicho análisis supone una importante ayuda no sólo en la toma de decisiones de política económica, sino también constituye un requisito indispensable y previo a las tareas de predicción necesarias en un contexto empresarial.

El estudio de una economía puede abordarse desde muy diversas ópticas, una de las cuales es el enfoque input-output, el cual permite analizar conjuntamente las relaciones intersectoriales de una economía y su demanda agregada, con lo cual se dispone de un conocimiento integrado de la actividad económica.

Consideramos la teoría de redes como una importante "herramienta", a nuestro juicio poco explotada en el ámbito económico, que constituye un marco general de estudio dentro del cual podemos encuadrar el análisis input-output. Esta teoría permite simplificar el esquema de relaciones surgido entre los sectores de una economía y, por lo tanto, favorecer la comprensión del mismo. El objetivo del presente trabajo es analizar las relaciones interindustriales bajo la óptica de la teoría de las redes sociales y, al mismo tiempo, efectuar una comparación entre los resultados así obtenidos y los que se derivan de la aplicación de los métodos inputoutput clásicos.
\end{abstract}

\section{Introducción}

El análisis input-output es una importante herramienta en los estudios de economía $y$, en particular, de economía regional, ya que permite un conocimiento integrado de la misma al proporcionar información no sólo de las relaciones existentes entre los distintos sectores, sino también sobre su demanda agregada. El esquema contable recogido en toda tabla input-output refleja las interdependencias en la estructura productiva analizada a través del conjunto de relaciones intersectoriales consideradas.

El modelo clásico input-ouput de Leontief (1951) se ha empleado habitualmente en el análisis de la estructura de una economía, posibilitando la realización de simulaciones y predicciones sobre impactos externos en la estructura económica. La comprensión a partir del mismo de las relaciones intersectoriales, entendidas como

\footnotetext{
${ }^{1}$ Enviar correspondencia a: Ana Salomé García Muñiz (asgarcia@correo.uniovi.es); Carmen Ramos Carvajal (cramos@correo.uniovi.es).
} 
flujos de producción, ha constituido, además, uno de los pilares en los estudios de localización de ejes de crecimiento, necesarios en la planificación de toda política industrial coordinada.

A pesar de sus conocidas limitaciones (Uriel, 1997; Muñoz, 1994, Pulido y Fontela, 1993) existe un consenso generalizado sobre el adecuado comportamiento de los coeficientes clásicos de Chenery y Watanabe, Rasmussen o Streit, para el estudio de las transacciones intersectoriales y su interdependencia dentro de la estructura productiva en cuestión. Estas medidas aplicadas tradicionalmente ${ }^{2}$ permiten obtener una visión general de la economía regional o nacional, ya que informan sobre las relaciones directas e indirectas entre las ramas de actividad perfilando los sectores claves en el funcionamiento del entramado económico.

Frente a esta óptica clásica de análisis, planteamos en este trabajo una alternativa, cuanto menos enriquecedora, de estudio del marco input-output. Un nuevo enfoque basado en un instrumento, a nuestro juicio poco explotado aún en el ámbito económico, como es la teoría de redes sociales. Es apenas hace diez años, en torno a los años noventa, cuando los economistas han empezado a emplear esta técnica "conscientemente" ${ }^{3}$ en sus estudios de mercado (Rauch y Casella, 2001).

Este trabajo es un primer paso de un estudio más ambicioso, en el que pretendemos reformular el análisis estructural input-output a partir de la teoría de redes sociales.

La aplicación de esta teoría en el ámbito del análisis input-output presenta un gran potencial al integrar en su desarrollo cuestiones tan relevantes como las posiciones relativas de los sectores, su orientación o los senderos por donde circula la influencia económica dentro de la estructura considerada. Permite, por lo tanto, una notable simplificación del esquema de relaciones intersectoriales, ventajas que apenas han sido aprovechadas aún en este marco. Consideramos el trabajo de Morillas (1983), en el que se analizan las relaciones intersectoriales a partir de la teoría de grafos, como el antecedente de la aplicación de la disciplina de las redes sociales.

2 Estos coeficientes clásicos han sido frecuentemente utilizados en numerosos estudios, así por ejemplo, podemos señalar los trabajos de Artís y otros (1993), López y Pulido (1993), Pérez y Martínez (1995) o Domínguez y Prado (2000), entre otros.

${ }^{3}$ Anteriormente existían ya aplicaciones sobre organización industrial y teoría de juegos a nivel de empresas individuales. 
En este documento ofrecemos una alternativa a los indicadores clásicos (coeficientes de Rasmussen, Chenery-Watanabe y Streit, entre otros) en el estudio de ramas clave en la economía. Bajo la óptica de las redes, la determinación de la posición de los sectores en la estructura económica a través de medidas de centralidad, establecerá la influencia e importancia de los mismos en la configuración de las transacciones bajo un nuevo enfoque.

A continuación, se plantea tras la especificación de la red económica considerada, unos indicadores de centralidad sobre la estructura analizada y sus similitudes o divergencias con las medidas tradicionales. Se concluye con un breve resumen de los resultados obtenidos.

\section{Una tabla input-output como una red de relaciones intersectoriales}

El concepto de red social es sencillo, consiste en un conjunto de actores entre los que se establece una serie de vínculos. Pueden estar constituidas por un número más o menos amplio de actores y una o más clases de relaciones entre pares de elementos. En palabras de R. Hanneman ${ }^{4}$ :

Para entender acertadamente una red social es necesario realizar una descripción completa y rigurosa de la estructura de sus relaciones como punto de partida para el análisis.

En el análisis de una red se considera la estructura de las relaciones en las que cada actor se encuentra involucrado, es más, estos actores se describen a través de sus conexiones, las cuales se muestran tan relevantes como ellos mismos.

El estudio de una red social se efectúa fundamentalmente a partir de dos grandes grupos de técnicas estrechamente relacionadas: la teoría de grafos y la teoría de matrices. Ambas permiten representar y describir una red de una manera sistemática $y$, por lo tanto, posibilitarán una más sencilla aproximación al estudio de las mismas, así como clarificar determinados comportamientos o actitudes.

Una tabla input-output (TIO) es una descripción detallada, en términos monetarios, del proceso productivo y de los flujos de bienes y servicios existentes en un espacio geográfico concreto durante el período de un año. Constituye una matriz en la que se recogen los flujos entre diferentes sectores de una economía, es decir, las

4 Esta cita ha sido obtenida de la versión electrónica de Hanneman (2000): Introducción a los métodos de análisis de redes sociales, disponible en la dirección http://www.redes-sociales.net/ 
compras y ventas entre las distintas ramas. A partir de los valores que aparecen en la tabla se pueden conocer las similitudes y diferencias existentes entre los sectores. Es interesante determinar no sólo la importancia que tiene cada sector en la economía de una región, sino también cómo se relacionan las diferentes ramas productivas a través de los flujos de comercio. Como señala Porter (1987):

(...) aunque el entorno relevante es muy amplio y abarca tanto fuerzas sociales como económicas, el aspecto clave del entorno de la empresa es el sector o sectores industriales en los cuales compite. La estructura de un sector industrial tiene una fuerte influencia al determinar las reglas del juego competitivas así como las posibilidades estratégicas potencialmente disponibles para la empresa.

Dicho análisis implica un conocimiento profundo de las relaciones intersectoriales, esto es, de las denominadas ligazones específicas.

El enfoque de este trabajo y, que a nuestro juicio es novedoso, consiste en tratar las relaciones existentes entre los sectores de una economía, desde la óptica de las redes sociales, por ello consideramos a las ramas productivas como actores y las relaciones entre ellas estarán representadas por los flujos de mercancías.

Esta aproximación a las relaciones intersectoriales, plantea un esquema más real y completo de su funcionamiento, ya que como sugiere Grannovetter (1985) a través de su teoría de "embeddedness", toda acción económica no puede ser explicada únicamente a través de móviles individuales, sino que depende también de las relaciones que los individuos mantienen ${ }^{5}$. Siguiendo este razonamiento, la utilización de las redes sociales permite, de forma simplificada, un conocimiento en detalle del esquema de relaciones de un sector con su entorno, el capital social ${ }^{6}$ en el cual se desarrolla el entramado de sus estrategias empresariales con el resto de agentes económicos.

La red económica establecida se convierte en un cúmulo de oportunidades y limitaciones que influye sobre el comportamiento de los sectores. En este marco,

${ }^{5}$ Esta teoría explica como mecanismos informales de confianza y expectativas de comportamiento cooperativo surgen entre las relaciones empresariales y facilitan la transferencia de recursos entre agentes económicos (Uzzi, 2001).

6 Ver una introducción al concepto de capital social en Molina et.al. (2000b), la cual supone una adaptación del epígrafe existente, como cita el autor, en Molina (2000a). 
las propias transacciones de las ramas productivas afectan, consecuentemente, a la toma de decisiones de otros sectores, y como tal, la estructura global se ve también transformada. Existe, en suma, un papel interactivo entre la acción o transacciones empresariales realizadas por las ramas consideradas y la estructura, de tal forma que la estructura emergente de la red también influye en el comportamiento de la empresa (Giddens, 1984). El planteamiento de estas relaciones entre los agentes individuales y la estructura como un todo, permite un análisis detallado de las transacciones económicas a nivel micro y macroeconómico, a través de la metodología de las redes sociales.

\section{La consideración de los sectores clave bajo una doble perspectiva: clásica y de teoría de redes}

Un uso común del análisis input-output consiste en establecer qué ramas productivas se consideran como claves, es decir, presentan unos efectos más destacados en el seno del sistema económico regional, cuantificando la intensidad y la relevancia de las ligazones intersectoriales. Con este fin, tradicionalmente, se han venido aplicando los coeficientes de Rasmussen (1956) y Chenery-Watanabe (1958), los cuales a partir de concepto de "eslabonamiento", introducido por Hirschman (1958) en estudios de desarrollo, permiten agrupar los sectores en cuatro bloques. Para ello, se distinguen los eslabonamientos hacia atrás que se producen cuando una rama productiva utiliza bienes y servicios procedentes de otras; y los eslabonamientos hacia adelante, debidos a que los productos de un sector son utilizados por otros como bienes y servicios en la elaboración de sus productos.

A partir de la teoría de redes sociales es posible profundizar en el conocimiento de la estructura productiva de una región o nación, determinando aquellos sectores claves mediante la consideración de tres rasgos complementarios que amplían y complementan la visión clásica sobre las ramas polarizadoras del crecimiento. Estas características permiten analizar las relaciones interindustriales bajo un triple enfoque: los efectos totales de un sector sobre el conjunto de la economía, la rapidez con que un sector se relaciona con los demás y la importancia de éstos como elementos trasmisores dentro de la red. Dichos aspectos se recogen dentro del concepto genérico de centralidad, característica que permite analizar las propiedades estructurales y de localización de la red económica.

En la teoría de redes se considera un actor como importante, si presenta un mayor número de interrelaciones, bien directas o indirectas, con el resto de agentes en la red. En este sentido, las ramas productivas que mantienen mayores conexiones 
gozan de posiciones estructurales más ventajosas en la medida en que presentan un mayor grado relativo de acceso y control sobre los recursos existentes, siendo menos dependientes. A partir de esta noción se identifican los sectores que funcionan en el sistema económico regional a modo de encrucijada, constituyendo elementos conectores cruciales para el funcionamiento e interconexión económica.

Esta óptica de estudio, profundiza y enriquece el conocimiento de la estructura productiva. Plantearemos en este trabajo la determinación de estos rasgos bien a través de las denominadas medidas de centralidad, y alternativamente con técnicas input-output tradicionales.

Según la metodología de las redes, para detectar aquellos sectores con una posición más relevante dentro de la economía se puede aplicar, siguiendo a Friedkin (1991), tres medidas de centralidad denominadas efectos totales, efectos inmediatos y efectos mediativos.

A pesar de la variedad de indicadores existentes con análoga finalidad ${ }^{7}$, su elección se ha basado en la similitud de planteamiento con la metodología clásica inputoutput, lo cual facilita una posterior comparación.

Dentro de la óptica clásica podemos referirnos a los conocidos coeficientes de Rasmussen que permiten determinar aquellos sectores con unos efectos más importantes sobre el conjunto de los agentes económicos de la red, tal y como plantean los efectos totales.

Por otro lado, los coeficientes de Streit identifican aquellas ramas más intensamente relacionadas con el sistema económico, lo cual las convierte en actividades productivas fundamentales para la interconexión del funcionamiento de la red.

A continuación se expone por separado la identificación de cada una de las tres características mencionadas, bajo los dos enfoques considerados, la metodología clásica y la nueva óptica de la teoría de las redes sociales.

\subsection{Los efectos directos e indirectos}

La determinación de la intensidad y relevancia de las transacciones interindustriales, bien directas o indirectas, puede ser estimada a partir de los

7 Otros conocidos índices de centralidad son la intermediación, la cercanía, lejanía y el índice de poder de Bonacich, por ejemplo. 
coeficientes de Rasmussen o de los denominados, dentro de las medidas de centralidad, efectos totales.

\subsubsection{Los coeficientes de Rasmussen}

En el modelo de Leontief simplificado ${ }^{8}$ los elementos de la denominada matriz inversa de Leontief (I-A) ${ }^{-1}$ indican la cuantía en que debe aumentar la producción de un sector i-ésimo para que la demanda final de un sector j-ésimo se incremente en una unidad. A partir de los elementos de esta matriz se pueden obtener coeficientes que recojan la capacidad de generar o absorber crecimiento de los distintos sectores de la economía. Un primer paso en este análisis consiste en considerar las sumas de los elementos de cada fila y columna de dicha matriz, esto es, los denominados efectos absorción $\left(z_{i}\right.$. $)$ y difusión $\left(z_{. j}\right)$, respectivamente.

Los coeficientes definidos por Rasmussen (1956) se desarrollan sobre la base de estos efectos difusión y absorción y se obtienen calculando, en primer término, un promedio de dichos efectos en cada una de las ramas, para después expresar cada uno de estos promedios en relación a los efectos globales.

El coeficiente de Rasmussen calculado a partir del efecto difusión y conocido por índice de poder de difusión se define como:

8 Una tabla input-output recoge las relaciones existentes entre los diferentes sectores, asimismo proporciona información sobre la demanda final y los inputs primarios de la economía. Dado que se trata de una matriz podemos aproximarnos a su estudio a partir de las filas o de las columnas. En el primer caso, es decir, si consideramos las filas, y partiendo del modelo simplificado de Leontief, podemos escribir:

\section{$\mathbf{X}=\mathbf{A} \mathbf{X}+\mathbf{Y}$}

donde $\mathbf{X}$ representa el vector de outputs totales, A corresponde a la matriz de coeficientes técnicos cuyos elementos se obtienen dividiendo los flujos intersectoriales entre los totales de las columnas e $\mathbf{Y}$ representa la demanda final.

Por lo tanto, operando se tiene que:

$$
\mathbf{X}=(\mathbf{I}-\mathbf{A})^{-1} \mathbf{Y}
$$




$$
U_{. j}=\frac{\frac{1}{n} \sum_{i=1}^{n} z_{i j}}{\frac{1}{n^{2}} \sum_{i=1}^{n} \sum_{j=1}^{n} z_{i j}}
$$

Cuantifica en términos relativos la fuerza con que un sector productivo es capaz de "arrastrar" al conjunto de la economía.

Si nos hubiésemos apoyado en el efecto absorción, habríamos derivado el índice de sensibilidad de absorción cuya expresión, a partir la matriz de coeficientes de distribución ${ }^{9}$, es:

$$
U_{i .}=\frac{\frac{1}{n} \sum_{j=1}^{n} z_{i j}}{\frac{1}{n^{2}} \sum_{i=1}^{n} \sum_{j=1}^{n} z_{i j}}
$$

Permite medir en términos relativos el impacto que recibe un sector ante un crecimiento del total de ramas.

Sobre la base de estos resultados se establece una clasificación en torno a cuatro categorías.

a) Los denominados sectores clave de una economía son aquellos que presentan efectos de arrastre hacia adelante y hacia atrás superiores a la media.

b) Los sectores con efectos de arrastre hacia atrás mayores que la unidad.

c) Sectores con eslabonamientos hacia adelante sobre la media, ya que una parte elevada de sus productos son vendidos a otras que los utilizan como bienes intermedios generando así ligazones elevadas de oferta.

d) Los sectores independientes que presentan unos efectos de dispersión y absorción por debajo de la media.

\subsubsection{Los efectos totales}

En la teoría de redes sociales, los distintos efectos se determinan a partir de una matriz (A) en la que se recogen las interrelaciones de la red analizada, la cual en el ámbito de nuestro estudio, es la matriz de coeficientes input-output.

${ }^{9}$ Los coeficientes de distribución se obtienen dividiendo los elementos de la matriz de flujos intersectoriales entre el total de cada fila. 
Siguiendo a Friedkin (1990), la determinación de los efectos totales intersectoriales está básicamente relacionada con el número y longitud de los caminos existentes entre los distintos sectores a través de las relaciones productivas especificadas, de tal forma que ${ }^{10}$ :

$$
\begin{gathered}
\mathbf{V}=(\mathbf{I}-\alpha \mathbf{A})^{-1}(1-\alpha)=\left(\mathbf{I}+\alpha \mathbf{A}+\alpha^{2} \mathbf{A}^{2}+\alpha^{3} \mathbf{A}^{3}+\ldots\right)(1-\alpha) \\
0<\alpha<1
\end{gathered}
$$

donde $\alpha$ es una ponderación de las influencias intersectoriales que permite calibrar la capacidad de influencia entre sectores.

El aumento del número de pasos a través de los cuales dos sectores se pueden interrelacionar supone una disminución del impacto de sus transacciones, mientras que para igualdad de distancias el efecto ocasionado depende de la intensidad o fuerza de las relaciones existentes $\left(\alpha \mathrm{a}_{\mathrm{ij}}\right)$.

Partiendo del supuesto más sencillo de ausencia de polarización en la toma de decisiones de los agentes económicos y suponiendo que $\alpha$ tiende a la unidad $\mathbf{V}$ podría converger, según las características de la matriz, a $\mathbf{V}_{\mathrm{u}}$ tal que los efectos totales intersectoriales son constantes ${ }^{11}$ :

$$
\mathbf{V}_{\mathrm{u}}=\left[\begin{array}{ccc}
\mathrm{c}_{1} & \ldots & \mathrm{c}_{\mathrm{n}} \\
\ldots & \ldots & \ldots \\
\mathrm{c}_{1} & \ldots & \mathrm{c}_{\mathrm{n}}
\end{array}\right]
$$

${ }^{10}$ Esta matriz de efectos intersectoriales totales (V) cumple diversas propiedades entre las cuales podemos señalar que es una matriz estocástica por filas:

$$
\begin{aligned}
& 0 \leq \mathrm{v}_{\mathrm{ij}} \leq 1 \\
& \sum_{\mathrm{j}=1}^{\mathrm{n}} \mathrm{v}_{\mathrm{ij}}=1
\end{aligned}
$$

11 Esta circunstancia se produce en aquellos casos en los cuales la matriz considerada es conexa y regular, es decir en términos de grafos o cadenas de Markov, sus elementos se encuentran relacionados bien directa o indirectamente y alguna de las potencias de la matriz presenta únicamente elementos positivos. 
La base de los efectos totales se podría calcular bajo estas condiciones como ${ }^{12}$ :

$$
\mathbf{V}=\lim _{\alpha \rightarrow 1}(\mathbf{I}-\alpha \mathbf{A})^{-1}(1-\alpha)=\mathbf{A}^{\infty}=\mathbf{V}_{\mathrm{u}}
$$

Los efectos totales de un sector $\mathrm{j}$ en la red se recogen en la columna $\mathrm{j}$ de la matriz $\mathbf{V}$, de tal forma que el efecto total de centralidad, TEC, se define como:

$$
\mathrm{C}_{\mathrm{TEC}(\mathrm{j})}=\frac{\sum_{\mathrm{i}=1}^{\mathrm{n}} \mathrm{v}_{\mathrm{ij}}}{\mathrm{n}} \quad \forall \mathrm{i}, \mathrm{j}
$$

Es decir, se trata del promedio de los elementos de las columnas de la matriz $\mathbf{V}$, de forma que cuanto mayor sea este valor, mayor fuerza tendrán en el sector los efectos totales respecto al conjunto de la economía.

Complementariamente, razonando de forma análoga a partir de la matriz de coeficientes de distribución, se plantearía un indicador sobre los efectos totales de la red sobre un sector i-ésimo:

$$
\mathrm{C}_{\mathrm{TEC}(\mathrm{i})}=\frac{\sum_{\mathrm{j}=1}^{\mathrm{n}} \mathrm{v}_{\mathrm{ij}}}{\mathrm{n}} \quad \forall \mathrm{i}, \mathrm{j}
$$

Cuanto mayor sea, mayor será el efecto recibido por un sector ante cambios en la red.

Es relevante notar que todas las medidas señaladas, tanto las que proceden del enfoque clásico como las derivadas de la teoría de las redes sociales, se obtienen a partir de la matriz inversa de Leontief. Es más, podemos considerar los coeficientes de Rasmussen como un caso particular de los derivados de la teoría de redes, donde no se consideran ponderaciones, es decir, se trata de medidas que no tienen en cuenta el factor $\alpha$ que representa la influencia de los sectores.

También es preciso señalar que mientras el coeficiente de Rasmussen se encuentra normalizado al estar divididos por el total, no ocurre lo mismo con los efectos totales, sin embargo, la interpretación de ambas medidas es análoga.

De esta forma, queda demostrada la similitud directa entre ambas medidas en el caso más sencillo de ausencia de polarización y ponderación de las influencias intersectoriales tendente a la unidad. En un análisis más detallado se podría

\footnotetext{
${ }^{12}$ Ver dicha formulación en Friedkin y Johnsen (1990).
} 
plantear la estimación de este coeficiente de influencia social $(\alpha)$, e incluso su diferenciación entre sectores $\left(\alpha_{i}\right)$, con el fin de distinguir la propensión de una rama productiva a las influencias intersectoriales. Quedaría así establecido el patrón de toma de decisiones empresariales junto con sus elementos dinamizadores del sistema económico.

\subsection{La rapidez en la difusión}

La rapidez de transmisión de los efectos totales determinados es una característica interesante en la valoración de la implementación de políticas económicas y sus posibles efectos en el tiempo o en su propagación dentro de la red analizada. Este rasgo se determina, siguiendo la terminología de las redes sociales, a partir de los denominados efectos inmediatos $y$ no ha sido considerado en los estudios tradicionales de input-output, por lo que se trata de una aportación neta de la teoría de redes al análisis estructural.

La rapidez de difusión en la red, una vez obtenidos los efectos totales de un sector $j$, se puede determinar a través de la longitud media de las secuencias de sus transacciones económicas:

$$
\mathbf{M}=\left(\mathbf{I}-\mathbf{Z}+\mathbf{E} \mathbf{Z}_{\mathbf{d g}}\right) \mathbf{D}
$$

donde $\mathbf{D}$ es una matriz diagonal con elementos $d_{i i}=1 / c_{i}$, $\mathbf{E}$ representa una matriz (nxn) unitaria y $\mathbf{Z}$ es la denominada matriz fundamental cuya expresión es la que sigue:

$$
\mathbf{Z}=\left(\mathbf{I}-\mathbf{A}+\mathbf{A}^{\infty}\right)^{-1}
$$

tal que $\mathbf{A}^{\infty}$ coincidirá con la matriz $\mathbf{V}_{\mathrm{u}}$ la cual, recoge el estado estacionario del proceso analizado $\left(c_{1}, \ldots, c_{n}\right)$.

$\mathrm{Y} \mathbf{Z}_{\mathrm{dg}}$ es una matriz diagonal construida a partir de la definición de $\mathbf{Z}$.

La rapidez con que un sector se relaciona económicamente con otros, se expresa en las columnas respectivas de la matriz $\mathbf{M}$. El indicador de estos efectos inmediatos, IEC, se calcula así como el inverso de la media de las longitudes de las relaciones intersectoriales de una rama productiva $\mathrm{j}$ :

$$
\mathbf{C}_{\operatorname{IEC}(\mathrm{j})}=\left(\frac{\sum_{\mathrm{i}=1}^{\mathrm{n}} \mathrm{m}_{\mathrm{ij}}}{\mathrm{n}}\right)^{-1} \quad \forall \mathrm{i}, \mathrm{j}
$$


Donde $\mathrm{m}_{\mathrm{ij}}$ son los elementos de la matriz $\mathbf{M}$.

A medida que aumenta el valor de la medida expuesta, mayor será la rapidez con la cual se propaguen los efectos totales del sector considerado.

\subsection{Elementos conectores}

El último de los tres rasgos que se ha considerado definen un sector económico como clave, hace referencia a la importancia de sectores particulares como instrumentos de transmisión de los efectos totales. Son sectores que facilitan el funcionamiento e interconexión económica, vertebrando la interrelación de las distintas actividades productivas. Tales agentes económicos funcionan en el sistema a modo de encrucijada, constituyendo puntos clave para el desarrollo conjunto de la economía.

La cuantificación de la intensidad de estas transacciones industriales en el sistema económico ha constituido además una de las cuestiones recurrentes en la literatura de economía regional. Su cálculo permite calibrar, los efectos de arrastre en el desarrollo y evolución de la región, de tal forma, que aquellas ramas que aparecen como habitualmente interrelacionadas podrían llegar a formar complejos industriales y establecerse juntas en el espacio.

El estudio de la debilidad o fortaleza de las interrelaciones sectoriales puede ser planteado bajo una doble perspectiva: a través de los tradicionales coeficientes de Streit o bien a partir de los efectos mediativos derivados de la noción de centralidad.

\subsubsection{Coeficientes de Streit}

Los coeficientes de Streit (1969) permiten analizar, a partir de la matriz de transacciones intermedias $\left(\mathbf{X}^{\prime}\right)$, las relaciones sector a sector determinando el grado de interrelación entre dos ramas cualesquiera. Serán considerados sectores muy vinculados, aquellos a los que van o de los que proceden gran parte de los inputs y outputs mutuamente utilizados.

Con esta finalidad, los indicadores denominados ligazones específicas de oferta $\left(\operatorname{LEO}_{i j}\right)$ y de demanda $\left(\operatorname{LED}_{\mathrm{ij}}\right)$ para los sectores $i$ y $j$ muestran, respectivamente, la proporción en la que la rama i utiliza los productos de la rama $j$ como consumo, o los usos intermedios que la rama $j$ hace de los productos de la rama $i$ con respecto al total de inputs intermedios (Muñoz, 1995): 


$$
\begin{aligned}
& \operatorname{LEO}_{\mathrm{ij}}=\frac{\mathrm{x}_{\mathrm{ij}}^{\prime}}{\sum_{\mathrm{j}=1}^{\mathrm{n}} \mathrm{x}_{\mathrm{ij}}^{\prime}} \\
& \operatorname{LED}_{\mathrm{ij}}=\frac{\mathrm{x}_{\mathrm{ij}}^{\prime}}{\sum_{\mathrm{i}=1}^{\mathrm{n}} \mathrm{x}_{\mathrm{ij}}^{\prime}}
\end{aligned}
$$

Cambiando el orden de los subíndices se pueden observar las otras dos relaciones entre el par de sectores analizados, esto es, $\sum_{\mathrm{i}=1}^{\mathrm{n}} \mathrm{x}_{\mathrm{ji}}^{\prime}, \sum_{\mathrm{j}=1}^{\mathrm{n}} \mathrm{x}_{\mathrm{ji}}^{\prime}$.

Este estudio de las ligazones se suele complementar con el cálculo de una medida resumen, formulada a partir de la media aritmética simple de las cuatro ligazones posibles existentes entre dos ramas cualesquiera $i$ y $j$. Formalmente, los denominados coeficientes simétricos de Streit $\left(\mathrm{CS}_{\mathrm{ij}}\right)$ toman la expresión siguiente:

$$
\mathrm{CS}_{\mathrm{ij}}=\frac{1}{4}\left[\mathrm{x}_{\mathrm{ij}}^{\prime}\left(\frac{1}{\sum_{\mathrm{j}=1}^{\mathrm{n}} \mathrm{x}_{\mathrm{ij}}^{\prime}}+\frac{1}{\sum_{\mathrm{i}=1}^{\mathrm{n}} \mathrm{x}_{\mathrm{ij}}^{\prime}}\right)+\mathrm{x}_{\mathrm{ji}}^{\prime}\left(\frac{1}{\sum_{\mathrm{j}=1}^{\mathrm{n}} \mathrm{x}_{\mathrm{ji}}^{\prime}}+\frac{1}{\sum_{\mathrm{i}=1}^{\mathrm{n}} \mathrm{x}_{\mathrm{ji}}^{\prime}}\right)\right]
$$

Su análisis permite perfilar los sectores productivos relevantes o polarizadores en el funcionamiento del sistema económico, dada su importancia como demandantes de los productos de otras ramas o como suministradores de inputs intermedios necesarios en la producción de otros sectores.

La posterior identificación de aquellos sectores más intensamente relacionados, no ya con otra rama determinada, sino con el conjunto del sistema económico, se estima a través del coeficiente de ligazón global $\left(\mathrm{CSG}_{\mathrm{i}}\right)$ :

$$
\mathrm{CSG}_{\mathrm{i}}=\sum_{\mathrm{j}=1}^{\mathrm{n}} \mathrm{CS}_{\mathrm{ij}}
$$

Estas ramas intensamente relacionadas, suponen actividades productivas fundamentales para la interconexión del funcionamiento de la economía considerada. Son sectores que funcionan a modo de encrucijada o transmisor dentro de la red. Interpretación idéntica a la de los denominados efectos mediativos.

\subsubsection{Efectos mediativos}


La matriz $\mathbf{M}$, planteada con anterioridad, se puede descomponer en el número de pasos desde un sector $j$ a otro $i$, a través de otros intermedios:

$$
\mathrm{m}_{\mathrm{ij}}=\sum_{\mathrm{k}=1}^{\mathrm{n}} \mathrm{t}_{(\mathrm{j}) \mathrm{jk}} \quad \mathrm{i} \neq \mathrm{j} \neq \mathrm{k}
$$

Donde $t_{(j) i k}$ es la ik- ésima celda de la matriz $\mathbf{T}$ en:

$$
\mathbf{T}_{(j)}=\left(\mathbf{I}-\mathbf{A}_{(j)}\right)^{-1}
$$

Y $\mathbf{A}_{(j)}$ es la matriz resultante de eliminar la j-ésima fila y columna de la matriz $\mathbf{A}$.

Los efectos mediativos indican la importancia de un sector j-ésimo como trasmisor o como punto de encrucijada para la conexión de la red económica:

$$
\mathrm{C}_{\mathrm{MEC}(\mathrm{j})}=\frac{\sum_{\mathrm{k}=1}^{\mathrm{n}} \overline{\mathrm{t}}_{(\mathrm{k}) \mathrm{j}}}{\mathrm{n}}
$$

Donde,

$$
\overline{\mathrm{t}}_{(\mathrm{k}) \mathrm{j}}=\frac{\sum_{\mathrm{i}=1}^{\mathrm{n}} \mathrm{t}_{(\mathrm{k}) \mathrm{ij}}}{(\mathrm{n}-1) \mathrm{t}_{(\mathrm{k}) \mathrm{jj}}} \mathrm{i} \neq \mathrm{j}
$$

recoge la contribución de un sector j-ésimo en trasmitir los efectos intersectoriales de la rama $k$.

El objetivo común de ambas técnicas consiste en cuantificar la relevancia de las ligazones intersectoriales de estas dos medidas presentadas, aunque el planteamiento matemático difiera notablemente. Sin embargo, los efectos mediativos permiten un análisis más completo que los coeficientes de Streit, ya que no sólo recogen las relaciones directas entre los diferentes sectores sino también las indirectas. Se trata, por tanto, de un indicador más rico sobre la intensidad de las transacciones totales. La consideración de los efectos inmediatos permitirá añadir además al estudio cuestiones sobre la rapidez de difusión de los efectos totales considerados, a través de la longitud media de las secuencias de sus transacciones económicas. Rasgos esenciales para profundizar en el conocimiento de los impactos de una política económica.

\section{Análisis de caso: estudio estructural de la economía asturiana}

La comparación de los resultados obtenidos al analizar las relaciones interindustriales de una determinada región o país, bajo la óptica de la teoría de las 
redes sociales y la aplicación de los métodos input-output clásicos, ofrece una visión práctica del grado de similitud de las técnicas planteadas.

En este trabajo, hemos desarrollado con esta finalidad, el estudio de la estructura económica asturiana a partir la matriz de Asturias correspondiente al 2000, por tratarse ésta de la última publicada ${ }^{13}$, a un nivel de desagregación de 16 sectores. Además, hemos considerado los coeficientes totales, ya que así se tienen en cuenta tanto las relaciones interiores como los flujos de importación.

En la determinación de la relevancia de las transacciones intersectoriales, bien directas o indirectas, tradicionalmente los coeficientes de Rasmussen establecen una clasificación en cuatro categorías de los sectores analizados. En el caso de la economía asturiana los resultados son mostrados en el siguiente Cuadro:

Cuadro No1. Coeficientes de Rasmussen

\begin{tabular}{|c|c|c|}
\cline { 2 - 3 } \multicolumn{1}{c|}{} & $\mathrm{U}_{\mathrm{j} j}>1$ & $\mathrm{U}_{. \mathrm{j}}<1$ \\
\hline $\mathrm{U}_{\mathrm{i} .}>1$ & I. Sectores clave & $\begin{array}{c}\text { II. Sectores con arrastre hacia } \\
\text { adelante }\end{array}$ \\
$\mathrm{U}_{\mathrm{i} .}<1$ & $\begin{array}{c}\text { III. Sectores con arrastre hacia } \\
\text { atrás }\end{array}$ & IV. Sectores independientes \\
& (3) (5) (6) (9) (10) & \\
\hline
\end{tabular}

Fuente: Elaboración propia a partir TIOA-2000.

Se denominan sectores claves de una economía a aquellos que presentan efectos de arrastre hacia delante y hacia atrás superiores a la media, entre ellos podemos señalar a los sectores Industria química (4), Industria transformadora de los metales (7) y Otras industrias manufactureras (8). Aquellas ramas que pueden ser consideradas como "impulsoras importantes de la economía regional" al gozar de fuertes efectos de arrastre hacia delante, son Agricultura y pesca (1) e Industrias extractivas (2). Sin embargo, las ramas Alimentación, bebidas y tabaco (3), Otros productos minerales no metálicos (5), Metalurgia y productos metálicos (6), Energía eléctrica, gas y agua (9) y Construcción (10), dados los elevados efectos de otros sectores sobre las mismas, pueden llegar a generar posibles

13 Para llevar a cabo este análisis utilizaremos la información recogida en la TIO de Asturias correspondiente al año 2000. Véase SADEI (2003, pendiente de publicación). Disponible electrónicamente en la página web de la Sociedad Asturiana de Estudios Económicos e Industriales (www.sadei.es). 
estrangulamientos del sistema económico, lo que las sitúa como ramas estratégicas para el desarrollo económico. Y por último, los sectores que no se encuadran en ninguna de las tres categorías anteriores y que se denominan independientes, son sectores poco importantes dado que ni provocan efectos de arrastre en la economía, ni reaccionan de forma significativa ante variaciones de la demanda de los agentes considerados. Si consideramos la distribución porcentual de los sectores en las categorías consideradas, se puede apreciar que los sectores estratégicos representa un $31 \%$ del total, los impulsores un $13 \%$ y los claves un $19 \%$, tal y como queda reflejado en el Gráfico №1.

Gráfico No1. Distribución sectorial

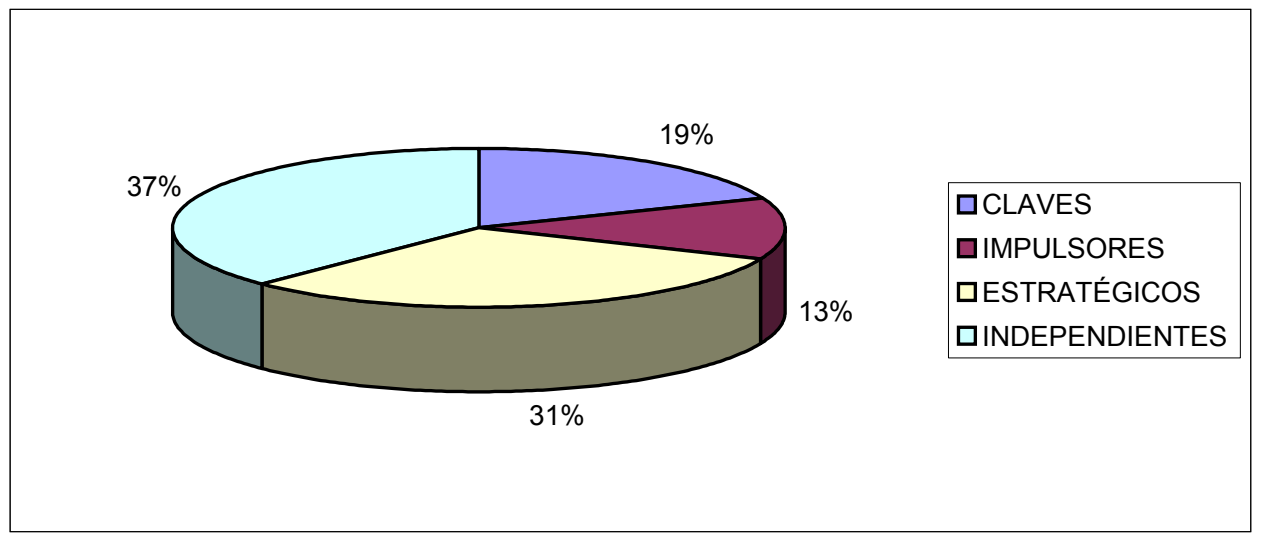

Fuente: Elaboración propia a partir de TIOA-2000.

La óptica aportada por la teoría de las redes sociales, permite estimar estos efectos totales de las relaciones intersectoriales directas e indirectas, a través de diversas medidas posicionales. El Cuadro No 2, expuesto a continuación, muestra lo resultados de este indicador junto con las otras dos medidas de centralidad desarrolladas por Friedkin (1990), los efectos inmediatos y los efectos mediativos:

Cuadro No2. Medidas de centralidad

\begin{tabular}{|c|c|c|c|c|}
\hline & \multicolumn{2}{|c|}{ Efectos totales } & Efectos inmediatos & Efectos mediativos \\
\hline Sectores & $\mathbf{C}_{\text {TEC (i) }}$ & $\mathbf{C}_{\text {TEC (j) }}$ & $\mathbf{C}_{\text {IEC (j) }}$ & $\mathbf{C}_{\text {MEC(j) }}$ \\
\hline $\mathbf{1}$ & 0,032 & 0,029 & 0,013 & 0,322 \\
\hline $\mathbf{2}$ & 0,017 & 0,089 & 0,082 & 0,630 \\
\hline $\mathbf{3}$ & 0,076 & 0,015 & 0,009 & 0,254 \\
\hline $\mathbf{4}$ & 0,017 & 0,081 & 0,062 & 0,582 \\
\hline $\mathbf{5}$ & 0,013 & 0,022 & 0,018 & 0,343 \\
\hline $\mathbf{6}$ & 0,161 & 0,065 & 0,044 & 0,505 \\
\hline $\mathbf{7}$ & 0,054 & 0,068 & 0,050 & 0,538 \\
\hline $\mathbf{8}$ & 0,023 & 0,063 & 0,040 & 0,497 \\
\hline $\mathbf{9}$ & 0,028 & 0,049 & 0,040 & 0,497 \\
\hline $\mathbf{1 0}$ & 0,206 & 0,073 & 0,053 & 0,549 \\
\hline $\mathbf{1 1}$ & 0,048 & 0,042 & 0,040 & 0,501 \\
\hline $\mathbf{1 2}$ & 0,058 & 0,009 & 0,008 & 0,235 \\
\hline $\mathbf{1 3}$ & 0,040 & 0,077 & 0,062 & 0,577 \\
\hline
\end{tabular}




\begin{tabular}{|c|c|c|c|c|}
\hline $\mathbf{1 4}$ & 0,110 & 0,307 & 0,126 & 0,671 \\
\hline $\mathbf{1 5}$ & 0,079 & 0,002 & 0,002 & 0,063 \\
\hline $\mathbf{1 6}$ & 0,037 & 0,010 & 0,009 & 0,236 \\
\hline $\begin{array}{c}\text { Promedi } \\
\text { o }\end{array}$ & 0,063 & 0,063 & 0,041 & 0,438 \\
\hline
\end{tabular}

Fuente: Elaboración propia a partir de TIOA-2000.

Obsérvese que si se decide extrapolar la catalogación planteada por los coeficientes de Rasmussen, la distribución porcentual de la categorías -Gráfico No 1- se mantiene, si bien el reparto subyacente difiere notablemente. La existencia de un factor corrector $(\alpha)$ de la capacidad de influencia intersectorial recogida a través de los coeficientes input-output, se muestra lógicamente como un elemento perturbador que permitiría recoger la sensibilidad de los sectores a la posición estructural desarrollada por los mismos, aspecto no considerado hasta ahora en este sentido en el análisis clásico input-output.

Si nos centramos en los efectos desarrollados por las ramas j-ésimas, los sectores clave bajo la perspectiva tradicional, esto es, Industria química (4), Industria transformadora de los metales (7) y Otras industrias manufactureras (8), presentan análogamente unos elevados efectos en el conjunto de los tres indicadores analizados. Su posición de centralidad en la red económica, les permite trasmitir unos importantes efectos totales sobre el conjunto de la economía con relativa rapidez, jugando un papel clave en la intermediación de las relaciones intersectoriales del resto de agentes productivos. Estas características se extienden además básicamente al siguiente conjunto de ramas productivas: Industrias extractivas (2), Metalurgia y productos metálicos (6), Construcción (10), Transporte y comunicaciones (13) y Servicios financieros y empresariales (14). En contraposición, los sectores Comercio y reparación (11), Hostelería (12), Educación, sanidad y servicios sociales (15) y Otros servicios (16) -sectores independientes en el Cuadro N01- poseen una posición menos central y por tanto, unos menores efectos posibles sobre la red económica configurada.

El principio de transmisión desarrollado a través del último de estos indicadores de centralidad considerados -efectos mediativos- constituye una cuestión clave para el desarrollo conjunto de una economía, compartida por los denominados coeficientes de Streit. 
A partir del Cuadro No 3, se puede determinar una ordenación jerárquica de las actividades productivas en función de la intensidad de su acción polarizadora estimada a través de los mencionados coeficientes de Streit ${ }^{14}$.

\section{Cuadro No 3. Coeficiente de Ligazón Global}

\begin{tabular}{|l|c|}
\hline \multicolumn{1}{|c|}{ Sectores } & CSGi \\
\hline Agricultura y pesca (1) & 0,904 \\
\hline Industrias extractivas (2) & 0,942 \\
\hline Alimentación, bebidas y tabaco (3) & 1,127 \\
\hline Industria química (4) & 0,999 \\
\hline Otros productos minerales no metálicos (5) & 0,727 \\
\hline Metalurgia y productos metálicos (6) & 1,319 \\
\hline Industria transformadora de los metales (7) & 0,982 \\
\hline Otras industrias manufactureras (8) & 0,909 \\
\hline Energía eléctrica, gas y agua (9) & 0,951 \\
\hline Construcción (10) & 1,275 \\
\hline Comercio y reparación (11) & 0,950 \\
\hline Hostelería (12) & 0,769 \\
\hline Transporte y comunicaciones (13) & 1,001 \\
\hline Servicios financieros y empresariales (14) & 1,585 \\
\hline Educación, sanidad y servicios sociales (15) & 0,842 \\
\hline Otros servicios (16) & 0,718 \\
\hline \multicolumn{1}{|c|}{ Promedio } & 1 \\
\hline
\end{tabular}

Fuente: Elaboración propia a partir de TIOA-2000.

Por encima del nivel medio, se sitúan Alimentación, bebidas y tabaco (3), Metalurgia y productos metálicos (6), Construcción (10), Transporte y comunicaciones (13) y Servicios financieros y empresariales (14), ramas que, a excepción de la primera, presentan análogamente efectos mediativos elevados. El resto de sectores con efectos trasmisores significativos, tales como Industrias extractivas (2), Industria química (4), Industria transformadora de los metales (7) y Otras industrias manufactureras (8), se localizan muy próximas a este punto de corte medio.

De esta forma, con fundamentos y objetivos comparables a las técnicas clásicas, queda establecida empíricamente la similitud entre ambas metodologías, si bien la incorporación de los conceptos desarrollados bajo la teoría de las redes sociales permiten ampliar el espectro del estudio estructural desarrollado. La posible

${ }^{14}$ Dado que los coeficientes de Streit se estiman como una media de proporciones relativas o, ligazones específicas, sin ponderación de ningún tipo, el coeficiente de ligazón global no debe interpretarse como una medida de la importancia cuantitativa de los complejos industriales, sino como un indicador cualitativo de su coherencia interna. 
estimación de la ponderación a aplicar en la estimación de los efectos totales $(\alpha)$, la rapidez a través de la cual se difunden los efectos totales, y la inclusión de las relaciones indirectas en la estimación de la transmisión de los mismos, son aspectos no considerados en la concepción tradicional de la metodología inputoutput clásica, que resultan de interés en el universo económico planteado.

\section{Conclusiones}

El análisis de los rasgos estructurales de una economía es un aspecto fundamental para la comprensión de su funcionamiento. La teoría de las redes sociales muestra una gran potencialidad en este sentido, al permitir una notable simplificación del esquema de relaciones intersectoriales contenido en una tabla input-output. Su utilización posibilita un conocimiento en detalle de las relaciones de un sector con su entorno, en el cual desarrolla el entramado de sus estrategias empresariales con el resto de agentes económicos.

Un aspecto importante en los estudios económicos es el análisis estructural. Pretendemos que este documento sea el primer paso en la reformulación del análisis estructural input-output a partir de la teoría de las redes sociales y, para ello, proponemos una visión alternativa de los indicadores clásicos en el estudio de ramas clave en la economía. A partir de la óptica de las redes, la determinación de la posición de los sectores en la estructura económica a través de medidas de centralidad, establece la influencia e importancia de los mismos en la configuración de las transacciones bajo un nuevo enfoque enriquecedor.

Con fundamentos y objetivos comparables a las técnicas clásicas, la consideración de tres rasgos complementarios para su estimación- efectos totales, inmediatos y mediativos - aporta características esenciales que permiten profundizar en el conocimiento del funcionamiento interno de la estructura económica analizada.

Frente a la metodología clásica, surge una nueva óptica que constituye una ampliación del esquema de relaciones observable en una tabla input-ouput. Si bien, los coeficientes clásicos de Rasmussen y los de Streit, se han empleado tradicionalmente para determinar, respectivamente, los sectores clave y las ramas fuertemente interrelacionadas, las medidas de centralidad muestran una capacidad superior para representar la realidad económica. No sólo aportan la rapidez de difusión de los efectos estimados, característica ausente en los indicadores tradicionales; además permiten una ponderación más exacta de las posibles influencias a través del coeficiente $\alpha$ en la expansión de los efectos totales, y la 
consideración de las relaciones indirectas, no incluidas en la estimación de los coeficientes de Streit, en la determinación de los sectores intensamente relacionados a través de los efectos mediativos.

\section{Bibliografía}

Álvarez, Rubén, García, A. S. y Ramos, C. (2001). "Análisis estructural de la economía asturiana: algunas alternativas", XV Reunión de ASEPELT España, La Coruña.

Artís, Manuel, Suriñach, J. y Pons, J. (1993). Caracterización de la industria catalana a partir de la tabla Input-Output de 1987, Documento de Trabajo, Departamento de Econometría, Estadística y Economía Española, Universidad de Barcelona.

Bonacich, Phillip (1987). "Power and centrality: A family of measures", American Journal of Sociology, Vol. 92, no 5, pp. 1170-1182.

Burt, Ronald. (1987). "Social Contagion and Innovation: Cohesion versus Structural Equivalence", American Journal of Sociology, Vol. 92, nº 5, pp. 1287-1335.

Chenery, Hollis. y Watanabe, T. (1958). "International comparisons of the structure of production". Econometrica, no 4, Vol. 26.

Domínguez, José María y Prado, C. (2000). Articulación interna de la economía vasca en el período 1990-1995, Informe no 1171, EUSTAT.

Freeman, Linton. (1978/79). "Centrality in Social Networks. Conceptual Clarification", Social Networks, no 1, pp. 215-239.

Friedkin, Noah. (1991). "Theoretical Foundations for Centrality Measures", American Journal of Sociology, Vol. 96, no 6, mayo, pp.1478-1504.

Friedkin, Noah y Johnsen, E. (1990). "Social Influence and Opinions", Journal of Mathematical Sociology, Vol. 15, no 3-4, mayo, pp.193-205.

García, Ana Salomé y Ramos, C. (2001). "Análisis de la realidad económica asturiana: un enfoque desde la teoría de grafos", XXVII Reunión de Estudios Regionales, Madrid.

García, Ana Salomé, Muñiz, M. y Ramos, C. (2002). "Análisis de la estructura productiva regional desde la óptica de la equivalencia estructural", XXVIII Reunión de Estudios Regionales, Murcia.

Giddens, Anthony (1984). The Constitution of Society: Outline of the Theory of Structuration. University of California Press, Berkeley.

Grannovetter, Mark (1985). "Economic action and social structure: The problem of embeddedness", American Journal of Sociology, Vol. 78, n 6, pp. 1360-1380.

Hanneman, Robert (2000). Introducción a los métodos de análisis de redes sociales, disponible en versión electrónica en http://www.redes-sociales.net/

Hirschman, Albert (1958). The Strategy of Economic Development. Yale University Press, New Haven.

Leontief, Wassily. (1951). The structure of the American Economy 1919-1929. Oxford University Press, New York.

López, Ana María y Pulido, A. (1993). "Análisis de las interrelaciones sectoriales en España", Economía Industrial, Marzo-Abril, pp. 167-178.

Molina, José Luis (2000a). El análisis de redes sociales. Aplicación al estudio de la cultura en las organizaciones. Tesis doctoral. Barcelona: UAB. 
Molina, José Luis, Muñoz, J. y Losego, P. (2000b). "Red y realidad: aproximación análisis de Redes científicas", VII Congreso Nacional de Psicología Social, Oviedo.

Molina, José Luis (2000c). El análisis de redes sociales. Una introducción. Barcelona, Edicions Bellaterra.

Morillas, Antonio (1983). La teoría de grafos en el análisis Input-Output. La estructura productiva andaluza. Editorial Universidad de Málaga, Málaga.

Muñoz, Candido (1988). "Elaboración y utilización de las tablas input-output regionales", Papeles de Economía Española, no 35, pp. 457-467.

Muñoz, Candido (1994). Las cuentas de la nación. Nueva Introducción a la Economía Aplicada. Editorial Civitas, Madrid.

Nohria, Nitin, y Eccles, R. (1992). Networks and Organizations. Structure, Form and Action. Harvard Business School Press, Boston, Mass.

Pardo, Ana y Rodríguez, P. (2000). "Sectores clave de la economía castellano leonesa 1995". Análisis input-output, XIV Reunión de ASEPELT España, Oviedo.

Pérez, Manuel A. y Martínez, S. (1995). "Industrias clave en la economía Asturiana. Análisis a través de las Tablas Input-Output de 1978, 1985 y 1990", Revista Asturiana de Economía, no 3, pp. 249-274.

Porter, Michael (1987). Estrategia competitiva. Técnicas para el análisis de los sectores industriales de la competencia. Compañía Editorial Continental, S.A., México.

Pulido, Antonio y Fontela, E. (1993). Análisis Input-Output. Modelos, Datos y Aplicaciones. Ediciones Pirámide, Madrid.

Rasmussen, P. Noregaard (1956). Studies in Intersectoral Relation. North Holland, Amsterdam.

Rauch, James y Casella, A. (2001). Networks and Markets. Russel Sage Foundation, New York.

SADEI (2003). Marco input-output de Asturias, disponible en versión electrónica en http://www.sadei.es

Streit, Manfred E. (1969). "Spatial Associations and Economic Linkages between industries", Journal of Regional Science, Vol.9, no 2, pp.177-88.

Uriel, Ezequiel. (1997). Contabilidad nacional. Ariel Economía, Barcelona.

Uzzi, Brian. (2001). "Knowledge Spillover in Corporate Financing Networks: Embeddedness, Network Transitivity and Trade Credit Performance", Strategic Management Journal, Vol.23, No 7, pp. 595-618.

Wasserman, Stanley. y Faust, K. (1994). Social Network Analysis. Methods and Applications, Structural Analysis in the Social Sciences. Cambridge University Press, New York. 